UUPHY $/ 97 / 10$

physics/9710040

\title{
Nonlinear Dirac and diffusion equations in $1+1$ dimensions from stochastic considerations
}

\author{
Karmadeva Maharana \\ Department of Physics, Utkal University, Bhubaneswar-751004, India
}

\begin{abstract}
We generalize the method of obtaining the fundamental linear partial differential equations such as the diffusion and Schrödinger equation, Dirac and telegrapher's equation from a simple stochastic consideration to arrive at certain nonlinear form of these equations. The group classification through one parameter group of transformation for two of these equations is also carried out.
\end{abstract}




\section{INTRODUCTION}

It is remarkable that some of the fundamental linear equations of physics such as the diffusion and Schrödinger equations, Dirac and telegrapher's equations, and the Maxwell equations can be obtained by setting up a master equation from simple stochastic considerations and a modification there of [1 3 ].

Hosts of nonlinear partial differential equations have been vigorously studied in recent times for possessing interesting solitonic, self focussing and allied properties in fields wide ranging from lasers to string theories. It is interesting to note, as it is shown in classical mechanics with randomness playing a key role, that any arbitrary initial distribution of velocities must eventually become Maxwellian. Thus the Maxwellian distribution must be invariant under the underlying stochastic process [4]. The system tending to and remaining in Maxwellian distribution is analogous to the self focussing in nonlinear optics and the properties of solitonic systems arising in nonlinear phenomena.

The transition to nonlinear equation from a linear equation through certain transformations is well known. An example is the Cole-Hopf transformation that carries over the linear diffusion equation to the nonlinear Burgers equation. The reverse process of getting linear

diffusion equation from a nonlinear diffusion equation in the form $\frac{\partial \phi}{\partial t}=v^{2} \frac{\partial^{2} \phi}{\partial v^{2}}$ has also been studied [8,9] through a nonlinear transformation.

In this paper, we propose to obtain a class of nonlinear equations in a different way by generalizing the method of [1]. The method is simple. The basic inputs can be incorporated from considerations and arguments based on physical reasoning to obtain nonlinear equations rather than arbitrary mathematical transformations. The form of the equations obtained are quite restrictive. However, we do not address the deep mathematical significance of Cole-Hopf transformation and the like in this method.

In section II, we briefly review the method used in setting up some basic linear equations of physics. Then we generalize the procedure of obtaining classes of correspondng nonlinear partial differential equations in section III. Next, section IV is devoted to the construction of the groups under which two of the equations obtained in section II, namely diffusion equation with nonlinearity and nonlinear telegrapher's equation, remain invariant. The similarity transformation and the Lie algebra are constructed to show the transformations under which solutions go over to new solutions. 


\section{LINEAR EQUATIONS}

The fundamental role of linear diffusion equation in Physics and its applications in other branches hardly needs any elaboration. An analytic continuation turns this equation into Schrödinger equation. Another basic equation of physics, the Dirac equation originated in the attempt to make Schrödinger equation compatible with special theory of relativity. It is a simple matter to arrive at telegrapher's equation by iterating Dirac equation in $(1+1)$ dimensions. It is a curiuos fact that these equations have been obtained by Gaveau etal [1] and Ord [2] from stochastic consideration by setting up a master equation. Ord [3] has also arrived at the Maxwell equation by a modification of the master equation.

Following [1] we briefly review how these equations are achieved and then proceed to nonlinear generalization. The basic consideration is the correlation over a random ensemble of particles. However, for simpler visualization we may follow the Boltzmann approach by analysing the movement of a single particle. Let a particle have random motion in one space dimension moving with a fixed speed $v$. We assume that it has complete reversal of direction of motion in a random manner from time to time, say as to flip of a coin. So this is according to Poisson distribution, that is to say that there is a fixed rate $a$ for this reversal and the probability for reversal in a time interval $d t$ is $a d t$. Let $P_{+}(x, t)$ (respectively, $\left.P_{-}(x, t)\right)$ be the probability density for the particle being at $x$ at time $t$ and moving to the right (respectively left). The master equation for an infinitesimal time step is

$$
P_{ \pm}(x, t+\Delta t)=P_{ \pm}(x \mp \Delta x, t)(1-a \Delta t)+P_{\mp}(x \pm \Delta x, t) a \Delta t
$$

This equation gives rise to the linear equations such as Dirac, telegrapher's, diffusion or Schrödinger equations in the lowest approximation under various circumstances.

To the lowest order in $\Delta x$ and $\Delta t$, equation (1) gives,

$$
\frac{\partial P_{ \pm}}{\partial t}=-a\left(P_{ \pm}-P_{\mp}\right) \mp v \frac{\partial P_{ \pm}}{\partial x}, \quad v=\frac{\Delta x}{\Delta t} .
$$

and the telegrapher's equation follows by iteration,

$$
\frac{\partial^{2} P_{ \pm}}{\partial t^{2}}-v^{2} \frac{\partial^{2} P_{ \pm}}{\partial x^{2}}=-2 a \frac{\partial P_{ \pm}}{\partial t}
$$

The one dimensional Dirac equation is obtained from (1) by analytic continuation. First

we identify $P_{ \pm}$with $u_{ \pm}, v \leftrightarrow c, \frac{i m c^{2}}{\hbar} \leftrightarrow a$, and then perform a phase transformation $u(x, t)=$ $e^{\frac{i m c^{2} t}{\hbar}} \Psi(x, t)$. This results in 


$$
i \hbar \frac{\partial \Psi}{\partial t}=m c^{2} \sigma_{x} \Psi-i c \hbar \sigma_{z} \frac{\partial \Psi}{\partial x} .
$$

In the Feynman path integral formulation through checkers moves on space-time, 1 has to be replaced by a factor $1+\left(\frac{i m c^{2}}{\hbar}\right) \Delta t$ for each step on which a reversal does not take place, whereas for reversals there is a factor $-i \Delta t \frac{m c^{2}}{\hbar}$.

The Dirac equation in $(1+1)$ dimensions, having two components, has the similar time and space dependence in this stochastic approach. But for a single component object we find that the linear diffusion equation results, which shows the asymmetry in derivatives arising out of the random walk problem.

A generalization to three space dimensions has been carried out in [1].

McKeon and Ord [5] have shown that if movements backward and forward in time is superposed as well on the previous motion, then Dirac equation in one dimension results without recourse to direct analytic continuation.

To arrive at the linear diffusion equation in a simple way, we put $P_{ \pm}=P_{\mp}=P$, and $a=\frac{1}{2 \Delta t}$. The master equation (1) reduces to

$$
P(x, t+\Delta t)=\frac{1}{2} P(x-\Delta x, t)+\frac{1}{2} P(x+\Delta x, t) .
$$

Expanding this in a Taylor series about the point $(x, t)$ gives,

$$
P(x, t)+\frac{\partial P(x, t)}{\partial t} \Delta t+\ldots=P(x, t)+\frac{\partial^{2} P(x, t)}{\partial x^{2}} \frac{(\Delta x)^{2}}{2}+\ldots
$$

and equating the lowest order terms we get,

$$
\frac{\partial P}{\partial t}=\frac{\partial^{2} P}{\partial x^{2}} \frac{(\Delta x)^{2}}{2 \Delta t}=D \frac{\partial^{2} P}{\partial x^{2}}
$$

where, $D=\frac{(\Delta x)^{2}}{2 \Delta t}$.

It may be noted that the above equation in the context of Brownian motion can be obtained from a consideration of one dimensional random walk with a Bernoulli distribution of probability and the statistical considerations sets [4]

$$
\lim _{\Delta t \rightarrow 0} \frac{(\Delta x)^{2}}{2 \Delta t}=D
$$

where $D$ is a constant.

A formal analytic continuation $(e . g . t \rightarrow i t$, or $D \rightarrow i \hbar)$ leads to Schrödinger equation for free particles. A potential $V(x, t)$ can be included by adding a term $V(x, t) P(x, t) \Delta t$ to the right hand side of equation (5). 
Ord [3] has obtained the Maxwell equations in $1+1$ dimensions by a modification of the master equation. We follow his procedure to show how it is done. First equation (1) is modified to,

$$
P_{ \pm}(x, t+\Delta t)=P_{ \pm}(x \mp \Delta x, t)+a(x, t) \Delta t
$$

where, $a(x, t)$ is interpreted as a source and linear combinations of $P_{+}$and $P_{-}$will correspond to the potentials $A(x, t)$ and $\Phi(x, t)$. To the lowest order in $\Delta x$ and $\Delta t$ equation (9) gives,

$$
\frac{\partial P_{ \pm}(x, t)}{\partial t} \Delta t=\mp \frac{\partial P_{ \pm}}{\partial x} \Delta x+a(x, t) \Delta t
$$

Writing,

$$
\begin{aligned}
& A(x, t)=\frac{1}{2}\left[P_{+}(x, t)+P_{-}(x, t)\right] \\
& \Phi(x, t)=\frac{1}{2}\left[P_{+}(x, t)-P_{-}(x, t)\right]
\end{aligned}
$$

equation (10) implies,

$$
\begin{aligned}
& \frac{\partial A(x, t)}{\partial t}=-c \frac{\partial \Phi(x, t)}{\partial x}+a(x, t) \\
& \frac{\partial \Phi(x, t)}{\partial t}=-c \frac{\partial A(x, t)}{\partial x}
\end{aligned}
$$

where we have put $\frac{\Delta x}{\Delta t}=c$.

Equations (10) and (11) may be decoupled by differentiating the first with respect to $t$ and the second with respect to $x$ to give ,

$$
\frac{\partial^{2} A(x, t)}{\partial t^{2}}=c^{2} \frac{\partial^{2} A(x, t)}{\partial x^{2}}+\frac{\partial a(x, t)}{\partial t}
$$

and similarly we get,

$$
\frac{\partial^{2} \Phi(x, t)}{\partial t^{2}}=c^{2} \frac{\partial^{2} \Phi(x, t)}{\partial x^{2}}-c \frac{\partial a(x, t)}{\partial t}
$$

Equations (13), (14), and (15) are equivalent to Maxwell equations in $(1+1)$ dimensions, equation (13) being the Lorentz condition

$$
\frac{\partial A(x, t)}{\partial x}+\frac{1}{c} \frac{\partial \Phi(x, t)}{\partial t}=0
$$

In order to obtain the wave equation for the "vector potential" $A$, we write, 


$$
\frac{1}{c} \frac{\partial a(x, t)}{\partial t}=4 \pi J(x, t)
$$

and equation (14) becomes,

$$
\frac{\partial^{2} A(x, t)}{\partial x^{2}}-\frac{1}{c^{2}} \frac{\partial^{2} A(x, t)}{\partial t^{2}}=-\frac{4 \pi}{c} J(x, t)
$$

and similarly writing

$$
\frac{1}{c} \frac{\partial a(x, t)}{\partial x}=-4 \pi \rho(x, t)
$$

equation (15) becomes the wave equation for scalar potential $\Phi(x, t)$,

$$
\frac{\partial^{2} \Phi(x, t)}{\partial x^{2}}-\frac{1}{c^{2}} \frac{\partial^{2} \Phi(x, t)}{\partial t^{2}}=-4 \pi \rho(x, t) .
$$

The two definitions (17) and (19) imply that

$$
\frac{\partial J(x, t)}{\partial x}+\frac{\partial \rho(x, t)}{\partial t}=0
$$

which is the equation of continuity. These considerations may be generalized to three space dimensions.

The objective of the above long review is to stress the interesting fact that many of the fundamental linear equations of physics are obtainable from an elementary consideration of stochastic process. Of course, by no stretch of imagination, we may expect the whole of physics to follow from such a consideration.

\section{NONLINEAR EQUATIONS}

Nonlinear partial differential equations appear in all branches of physics and some of these have interesting properties such as soliton like solutions, infinite number of conserved objects and so on. The nonlinear diffusion equation in the form (in our notation)

$$
\frac{\partial P(x, t)}{\partial t}=\frac{\partial}{\partial x}\left[f(p) \frac{\partial P(x, t)}{\partial x}\right]
$$

is well known in literature [6-10] and the properties of its solutions have been extensively studied.

Now we proceed with an aim at getting the above nonlinear equation and others out of the master equation (1), by suitable modifications. If we consider this to be a phenomenological 
equation, without any recourse to Poisson's distribution, then the obvious way to introduce nonlinearity is to introduce functions of $x$ and $t$ as multiplicative coefficients on right hand side of equation (1). As an example, suppose we apply randomness to $\Delta x$ itself. We know that if $\alpha$ is chosen at random in the interval $(0,1)$ then the probability of $\alpha_{n}$ to be in the interval $(x, x+d x)$ is given by $\frac{d x}{(1+x) \log 2}$ for large $n$, and hence a possibility is to use $\frac{\Delta x}{(1+x) \log 2}$ instead of $\Delta x$ in equation (5), which is a special case of equation (1). Perhaps it would be simplest to replace $\Delta x$ by $P(x, t) \Delta x$ in (5), and the resulting equation is

$$
\frac{\partial P(x, t)}{\partial t}=D P^{2}(x, t) \frac{\partial^{2} P(x, t)}{\partial x^{2}}
$$

where

$$
D=\frac{(\Delta x)^{2}}{\Delta t}
$$

Or else, we may treat $x$ and $t$ in the same footing, that is set $\Delta t \rightarrow P(x, t) \Delta t$ and $\Delta x \rightarrow$ $P(x, t) \Delta x$ instead of only $\Delta x \rightarrow P(x, t) \Delta x$ and we get,

$$
\frac{\partial P(x, t)}{\partial t}=D P(x, t) \frac{\partial^{2} P(x, t)}{\partial x^{2}}
$$

both equations (23) and (25) being nonlinear equations. Henceforth we set $D=1$.

It should be noted that this does not mean any nonlinear equation can be obtained this way. The condition that for $\Delta t=0, \Delta x=0$ both left and right side of equation (1) must match is quite a restriction. However, by making use of equation (7) one may get many more equations by setting the source term as function of $x, P$ and its derivatives or their combinations. This would be analogous to adding terms to the Lagrangian arbitrarily in the conventional method of getting equations of motion.

It is an interesting fact that if the master equation (1) is modified in the first term of right hand side in the following way

$$
P_{ \pm}(x, t+\Delta t)=P_{ \pm}(x \mp \Delta x, t)\left(1-P_{+} \Delta t\right)+P_{\mp}(x \pm \Delta x, t) a \Delta t
$$

we get a nonlinear form of the Dirac equation in one space dimension,

$$
\begin{aligned}
& \frac{\partial P_{+}}{\partial t}=-P_{+}^{2}-v \frac{\partial P_{+}}{\partial x}+a P_{-} \\
& \frac{\partial P_{-}}{\partial t}=-P_{+} P_{-}+a P_{+}+v \frac{\partial P_{-}}{\partial x}
\end{aligned}
$$


and by iteration a nonlinear analogue of telegraphers' equation results,

$$
\frac{\partial^{2} P_{+}}{\partial t^{2}}-v^{2} \frac{\partial^{2} P_{+}}{\partial x^{2}}=-P_{+}^{3}+P_{+} \frac{\partial P_{+}}{\partial t}+v P_{+} \frac{\partial P_{+}}{\partial x}+a^{2} P_{+}
$$

Further generalizations would be to consider $P$ as a complex multi- component object and readers may amuse themselves by putting objects such as supersymmetric variables, Pauli and other matrices etc. as coefficients of $\Delta x$ in equation (1).

\section{GROUP ANALYSIS}

Before we analyze the nonlinear equation (23) or (25) we would like to emphasize the important developments regarding the linear diffusion equation (7). It is well known that this equation goes over to Burgers equation under the Cole-Hopf transformation. Burgers equation and similar integrable ones have been much studied in recent decades for their importance in physical sciences for the existence of soliton like behaviour, infinite number of conservation laws as well as for their deep mathematical connections to Bäcklund transformations, conformal invariance and so on.

Equations of the form (23) have been analysed by Munier etal [8] and by Hill [9] in detail. It is found that the nonlinear diffusion equation of the form

$$
\frac{\partial \psi}{\partial t}=\psi^{2} \frac{\partial^{2} \psi}{\partial P^{2}}
$$

are equivalent to the classical diffusion equation for $P$,

$$
\frac{\partial P}{\partial t}=\frac{\partial^{2} P}{\partial x^{2}}
$$

if we introduce $x$ such that

$$
\psi(P, t) \equiv \frac{\partial P}{\partial x}
$$

and every nonlinear diffusion equation of the form

$$
\frac{\partial P}{\partial t}=\frac{\partial}{\partial x}\left[f(P) \frac{\partial P}{\partial x}\right]
$$

can be transformed to the following equation with a simpler nonlinearity

$$
f(P) \frac{\partial \psi}{\partial t}=\psi^{2} \frac{\partial^{2} \psi}{\partial P^{2}}
$$


where $\psi(P, t)$ is the flux associated with equation (33). Hence, for this special case the analysis would be similar to that of the linear diffusion equation.

However, in general the simplest nonlinear equation we would get from the master equation by replacing $\Delta x \rightarrow f(P) \Delta x$ in equation (5) would be,

$$
\frac{\partial P}{\partial t}=f^{2}(P) \frac{\partial^{2} P}{\partial x^{2}}
$$

as in equation (23) or

$$
\frac{\partial P}{\partial t}=f(P) \frac{\partial^{2} P}{\partial x^{2}}
$$

as in equation (25).

Now we proceed to analyse the properties of the solutions of (35) by means of one parameter groups as in [6 10]. For the single dependent variable $P$ and for the two independent variables $x$ and $t$ we have one parameter groups of the form,

$$
\begin{aligned}
& x_{1}=f(x, t, P, \epsilon)=x+\epsilon \xi(x, t, P)+O\left(\epsilon^{2}\right), \\
& t_{1}=g(x, t, P, \epsilon)=t+\epsilon \eta(x, t, P)+O\left(\epsilon^{2}\right), \\
& P_{1}=h(x, t, P, \epsilon)=P+\epsilon \zeta(x, t, P)+O\left(\epsilon^{2}\right) .
\end{aligned}
$$

We follow the standard procedure [9,10] to obtain the similarity variable and functional form of the solution by solving the first order partial differential equation

$$
\xi(x, t, P) \frac{\partial P}{\partial x}+\eta(x, t, P) \frac{\partial P}{\partial t}=\zeta(x, t, P)
$$

for known functions $\xi(x, t, P), \eta(x, t, P)$ and $\zeta(x, t, P)$. Let

$$
\mathbf{v}=\xi(x, t, P) \frac{\partial}{\partial x}+\eta(x, t, P) \frac{\partial}{\partial t}+\zeta(x, t, P) \frac{\partial}{\partial P}
$$

be a vector field on the space $X \times U^{(2)}$, where coordinates represent the independent variables, the dependent variables and the derivatives of the dependent variables up to order 2. All possible coefficient functions $\xi, \eta, \zeta$ are to be determined so that the one parameter group $\exp (\epsilon \mathbf{v})$, thus obtained would be the symmetry group of the nonlinear equations (35) for the diffusion case and (29) for telegrapher's case.

The determining equation for the symmetry group for the diffusion with non linearity, equation (35) is 


\begin{tabular}{lll}
\hline monomial & coefficients & \\
\hline$\frac{\partial^{2} P}{\partial x \partial t} \frac{\partial P}{\partial x}$ & $\eta_{P}=0$ & $(\mathrm{~A})$ \\
$\frac{\partial^{2} P}{\partial x \partial t}$ & $\eta_{x}=0$ & $(\mathrm{~B})$ \\
$\left(\frac{\partial P}{\partial x}\right)^{3}$ & $\xi_{P P}=0$ & $(\mathrm{C})$ \\
$\left(\frac{\partial P}{\partial x}\right)^{2} \frac{\partial P}{\partial t}$ & $\eta_{P P}=0$ & $(\mathrm{D})$ \\
$\left(\frac{\partial P}{\partial x}\right)^{2}$ & $\left(\zeta_{P}-2 \xi_{x}\right)_{P}=0$ & $(\mathrm{E})$ \\
$\left(\frac{\partial P}{\partial t}\right)^{2}$ & $-\eta_{P}+\eta_{P}=0$ & $(\mathrm{~F})$ \\
$\left(\frac{\partial P}{\partial x}\right)^{\left(\frac{\partial P}{\partial t}\right)}$ & $-\xi_{P}=-2 \eta_{x P} f(P)-3 \xi_{P}$ & $(\mathrm{G})$ \\
$\frac{\partial P}{\partial x}$ & $-\xi_{t}=f(P)\left(2 \zeta_{x P}-\xi_{x x}\right)$ & $(\mathrm{H})$ \\
$\frac{\partial P}{\partial t}$ & $\eta_{t}=f(P) \eta_{x x}+2 \xi_{x}+\frac{f^{\prime}(P) \xi}{f(P)}$ & $(\mathrm{I})$ \\
$P^{0}$ & $\zeta_{t}-f(P) \zeta_{x x}=0$ & $(\mathrm{~J})$ \\
\hline
\end{tabular}

where prime denotes differentiation with respect to the argument and subscript denote differentiation with respect to the indicated variable. These equations turn out to be the same as those of the nonlinear diffusion equation of the form,

$$
\frac{\partial P}{\partial t}=\frac{\partial}{\partial x}\left[f(P) \frac{\partial P}{\partial x}\right]
$$

considered in Hill [9].

From $(A),(B)$, and $(G)$ it is easily seen that

$$
\begin{gathered}
\xi=\xi(x, t), \quad \eta=\eta(t) \\
\zeta_{P}=2 \xi_{x}+r,
\end{gathered}
$$

where $r$ is a constant. So

$$
\zeta_{P P}=0
$$

From $(I)$ we get,

$$
\zeta=\frac{f(P)}{f^{\prime}(P)}\left[2 \xi_{x}-\eta_{t}\right]
$$

so that either

$$
2 \frac{\partial \xi}{\partial x}=\frac{\partial \eta}{\partial t}
$$


or

$$
\left[\frac{f(P)}{f^{\prime}(P)}\right]_{P P}=0
$$

that is,

$$
f(P)=a(P+b)^{m}
$$

where $a, b$ and $m$ denote arbitrary constants. If equation (45) holds, then from $(h)$ and equation (44), we obtain,

$$
\begin{aligned}
& \xi(x, t, P)=\beta+\gamma x \\
& \eta(x, t, P)=2 \theta+2 \gamma t \\
& \zeta(x, t, P)=0
\end{aligned}
$$

where, $\beta, \theta$ and $\gamma$ are arbitrary constants.

Hence, the Lie algebra of infinitesimal symmetries of the equation is spanned by the three vector fields,

$$
\begin{aligned}
\mathbf{v}_{\mathbf{1}} & =\frac{\partial}{\partial x} \\
\mathbf{v}_{\mathbf{2}} & =\frac{\partial}{\partial t} \\
\mathbf{v}_{\mathbf{3}} & =x \frac{\partial}{\partial x}+2 t \frac{\partial}{\partial t},
\end{aligned}
$$

and the commutation relations are given by,

$$
\left[\mathbf{v}_{1}, \mathbf{v}_{2}\right]=0, \quad\left[\mathbf{v}_{1}, \mathbf{v}_{3}\right]=\mathbf{v}_{1}, \quad\left[\mathbf{v}_{2}, \mathbf{v}_{3}\right]=2 \mathbf{v}_{2}
$$

The one parameter groups $G_{i}$ generated by the $\mathbf{v}_{i}$ are given below. The entries give the transformed points $\exp \left(\epsilon \mathbf{v}_{i}\right)(x, t, P)=\left(x_{1}, t_{1}, P_{1}\right)$.

$$
\begin{aligned}
& G_{1}:(x+\epsilon, t, P), \\
& G_{2}:(x, t+\epsilon, P), \\
& G_{3}:\left(e^{\epsilon} x, e^{2 \epsilon} t, P\right) .
\end{aligned}
$$

Each group $G_{i}$ is a symmetry group and if $P=q(x, t)$ is a solution of our nonlinear diffusion equation, so are the functions, 


$$
\begin{aligned}
& P^{(1)}=q(x-\epsilon, t), \\
& P^{(2)}=q(x, t-\epsilon), \\
& P^{(3)}=q\left(e^{-\epsilon} x, e^{-2 \epsilon} t\right) .
\end{aligned}
$$

The groups we obtain are the same as those for equations (40) and so is the similarity variable 9

$$
\omega=\frac{x+\alpha}{(t+\beta)^{1 / 2}}
$$

However, the functional form

$$
P=s(\omega)
$$

of the solution satisfies the ordinary differential equation

$$
2 f(s) \frac{d^{2} s}{d \omega^{2}}+\omega \frac{d s}{d \omega}=0
$$

whereas that corresponding to equation (40) is given by,

$$
2 f(s) \frac{d^{2} s}{d \omega^{2}}+2 \frac{d f(s)}{d s}\left(\frac{d s}{d \omega}\right)^{2}+\omega \frac{d s}{d \omega}=0 .
$$

In the case $f(P)$ is given by equation (46),

$$
\zeta=\left(\frac{P+b}{m}\right)\left[2 \frac{\partial \xi}{\partial x}-\frac{\partial \eta}{\partial t}\right]
$$

and for the time derivative of $\xi$ we get,

$$
\frac{\partial \xi}{\partial t}=f(P)\left[1-\frac{4}{m}\right] \frac{\partial^{2} \xi}{\partial x^{2}}
$$

while substituting (57) into $(J)$ and using (58) gives,

$$
\eta_{t t}=-\frac{8}{m} \xi_{x x x}
$$

So there are two possibilities arising out of equation (58), either for all constants $m$,

$$
\frac{\partial \xi}{\partial t}=\frac{\partial^{2} \xi}{\partial x^{2}}=\frac{\partial^{2} \eta}{\partial t^{2}}=0
$$

or for $m=4$,

$$
\frac{\partial \xi}{\partial t}=\frac{\partial^{3} \xi}{\partial x^{3}}=\frac{\partial^{2} \eta}{\partial t^{2}}=0
$$


Thus for all $m$ we have,

$$
\begin{aligned}
\xi(x, t, P) & =\mu+\sigma x, \\
\eta(x, t, P) & =\nu+\rho t, \\
\zeta(x, t, P) & =\left(\frac{P+b}{m}\right)(2 \sigma-\rho),
\end{aligned}
$$

where $\mu, \nu, \sigma$ and $\rho$ are arbitrary constants and the infinitesimal symmetries are spanned by four vector fields

$$
\begin{aligned}
& \mathbf{v}_{1}=\frac{\partial}{\partial x} \\
& \mathbf{v}_{2}=\frac{\partial}{\partial t} \\
& \mathbf{v}_{3}=x \frac{\partial}{\partial x}+\frac{2}{m}(P+b) \frac{\partial}{\partial P} \\
& \mathbf{v}_{4}=t \frac{\partial}{\partial t}-\frac{(P+b)}{m} \frac{\partial}{\partial P}
\end{aligned}
$$

and the commutation relations are given by,

$$
\left[\mathbf{v}_{1}, \mathbf{v}_{2}\right]=\left[\mathbf{v}_{1}, \mathbf{v}_{4}\right]=\left[\mathbf{v}_{2}, \mathbf{v}_{3}\right]=\left[\mathbf{v}_{3}, \mathbf{v}_{4}\right]=0, \quad\left[\mathbf{v}_{1}, \mathbf{v}_{3}\right]=\mathbf{v}_{1}, \quad\left[\mathbf{v}_{2}, \mathbf{v}_{4}\right]=\mathbf{v}_{2} .
$$

The one parameter groups $G_{i}$ generated by the $\mathbf{v}_{i}$ are,

$$
\begin{aligned}
& G_{1}:(x+\epsilon, t, P), \\
& G_{2}:(x, t+\epsilon, P), \\
& G_{3}:\left(e^{\epsilon} x, t,(P+b) e^{\frac{2 \epsilon}{m}}\right), \\
& G_{4}:\left(x, e^{\epsilon t},(P+b) e^{-\frac{\epsilon}{m}}\right),
\end{aligned}
$$

and if $P=y(x, t)$ is a solution to our non linear diffusion equation, so are the functions

$$
\begin{aligned}
& P^{(1)}=y(x-\epsilon, t, P), \\
& P^{(2)}=y(x, t-\epsilon, P), \\
& P^{(3)}=y\left(e^{-\epsilon} x, t,(P-b) e^{-\frac{2 \epsilon}{m}}\right), \\
& P^{(4)}=y\left(x, e^{-\epsilon} t,(P-b) e^{\frac{\epsilon}{m}}\right) .
\end{aligned}
$$

The similarity variable in this case is given by,

$$
\omega=\frac{x+\frac{\mu}{\sigma}}{\left(t+\frac{\nu}{\rho}\right)^{\frac{\sigma}{\rho}}}
$$


and the functional form of the solution is,

$$
P=\left(t+\frac{\nu}{\rho}\right)^{\left(\frac{2 \sigma}{m \rho}-1\right)} s(\omega)-b .
$$

Now for the nonlinear form of the telegrapher's equation (29), arising out of the nonlinear Dirac equation (27), the independent determining equations of the symmetry group are given below.

\begin{tabular}{lll}
\hline monomial & \multicolumn{1}{c}{ coefficient } \\
\hline$\frac{\partial^{2} P_{+}}{\partial t^{2}} \frac{\partial P_{+}}{\partial t}$ & $\eta_{P_{+}}=0$ & (a) \\
$\frac{\partial^{2} P_{+}}{\partial x \partial P_{+}} \partial t$ & $\xi_{P_{+}}=0$ & (b) \\
$\frac{\partial^{2} P_{+}}{\partial x \partial t}$ & $\xi_{t}=v^{2} \eta_{x}$ & (c) \\
$\frac{\partial^{2} P_{+}}{\partial t^{2}}$ & $\eta_{t}=\xi_{x}$ & \\
$\left.\frac{\partial P_{+}}{\partial t}\right)$ & $2 \zeta_{t P_{+}}-\eta_{t t}+v^{2} \eta_{x x}+P_{+}\left(\zeta_{P_{+}}-2 \xi_{x}\right)-$ \\
& $P_{+} v \eta_{x}-P_{+} \eta_{x}=0$ \\
$\frac{\partial P_{+}}{\partial x}$ & $\xi_{t t}+v^{2}\left(2 \zeta_{x} P_{+}-\xi_{x x}\right)+\zeta+$ \\
& $P_{+} v\left(\zeta_{P_{+}}-2 \xi_{x}\right)+P_{+}\left(\zeta_{P_{+}}-\xi_{x}\right)+$ \\
& $v P_{+}\left(\zeta_{P_{+}}-\xi_{x}\right)=0$ \\
& $\zeta_{t t}-v^{2} \zeta_{x x}-P_{+}^{3}\left(\zeta_{P_{+}}-2 \xi_{x}\right)+$ \\
$\left(P_{+}\right)^{0}$ & $P_{+} a^{2}\left(\zeta_{P_{+}}-2 \xi_{x}\right)+3 P_{+}{ }^{2} \zeta+P_{+} \zeta_{x}+$ \\
& $v \zeta_{x P_{+}}+a^{2} \zeta=0$ \\
\hline
\end{tabular}

The solutions are given by,

$$
\begin{aligned}
& \xi\left(x, t, P_{+}\right)=A v^{2} t+B, \\
& \eta\left(x, t, P_{+}\right)=A x+E, \\
& \zeta\left(x, t, P_{+}\right)=0,
\end{aligned}
$$

where, $A, B$, and $E$ are arbitrary constants and the infinitesimal symmetries are spanned by the three vector fields 


$$
\begin{aligned}
& \mathbf{v}_{1}=\frac{\partial}{\partial x}, \quad \text { space translation, } \\
& \mathbf{v}_{2}=\frac{\partial}{\partial t}, \quad \text { time translation, } \\
& \mathbf{v}_{3}=v^{2} t \frac{\partial}{\partial x}+x \frac{\partial}{\partial t}, \quad \text { hyperbolic "rotation" in } x, t \text { space, }
\end{aligned}
$$

with the commutation relations,

$$
\left[\mathbf{v}_{1}, \mathbf{v}_{2}\right]=0, \quad\left[\mathbf{v}_{1}, \mathbf{v}_{3}\right]=\mathbf{v}_{2}, \quad\left[\mathbf{v}_{2}, \mathbf{v}_{3}\right]=v^{2} \mathbf{v}_{1}
$$

The one parameter groups $G_{i}$ generated by the $\mathbf{v}_{i}$ are,

$$
\begin{aligned}
& G_{1}:\left(x+\epsilon, t, P_{+}\right), \\
& G_{2}:\left(x, t+\epsilon, P_{+}\right), \\
& G_{3}:\left(x+v^{2} \epsilon t, t+\epsilon x, P_{+}\right) .
\end{aligned}
$$

This implies that if $P_{+}=z(x, t)$ is a solution to equation (29), so are the functions

$$
\begin{aligned}
& P_{+1}=z(x-\epsilon, t), \\
& P_{+2}=z(x, t-\epsilon), \\
& P_{+3}=z\left(x-v^{2} \epsilon t, t-\epsilon x\right),
\end{aligned}
$$

where $\epsilon$ is any real number.

In order to compare the above vector fields of equation (70) with those of the linear second order form of the telegrapher's equation (3), we have the corresponging independent determining equations of the symmetry group:

\begin{tabular}{lll}
\hline monomial & coefficient & \\
\hline$\frac{\partial^{2} P_{+}}{\partial x^{2}} \frac{\partial P_{+}}{\partial x}$ & $\xi_{P_{+}}=0$ & $(\mathrm{P})$ \\
$\frac{\partial^{2} P_{+}}{\partial x^{2}} \frac{\partial P_{+}}{\partial t}$ & $\eta_{P_{+}}=0$ & $(\mathrm{Q})$ \\
$\frac{\partial^{2} P_{+}}{\partial x^{2}}$ & $\xi_{x}=\eta_{t}$ & $(\mathrm{R})$ \\
$\frac{\partial^{2} P_{+}}{\partial x \partial t}$ & $\xi_{t}=v^{2} \eta_{x}$ & $(\mathrm{~S})$ \\
$\left(\frac{\partial P_{+}}{p t}\right)^{2}$ & $\zeta_{P_{+} P_{+}}-2 \eta_{t P_{+}}+4 a \eta_{P_{+}}=0$ & $(\mathrm{~T})$ \\
$\frac{\partial P_{+}}{\partial x}$ & $\xi_{t t}-v^{2} \xi_{x x}+2 v^{2} \zeta_{x P_{+}}+2 a \zeta_{t}=0$ & $(\mathrm{U})$ \\
$\frac{\partial P_{+}}{\partial t}$ & $\eta_{t t}-v^{2} \eta_{x x}-2 \zeta_{t P_{+}}-2 a \eta_{t}=0$ & $(\mathrm{~V})$ \\
$\left(P_{+}\right)^{0}$ & $\zeta_{t t}-v^{2} \zeta_{x x}+2 a \zeta_{t}=0$ & $(\mathrm{~W})$ \\
\hline
\end{tabular}


The solutions are given by,

$$
\begin{aligned}
& \xi\left(x, t, P_{+}\right)=K v^{2} t+L, \\
& \eta\left(x, t, P_{+}\right)=K x+M, \\
& \zeta\left(x, t, P_{+}\right)=-K a x P_{+}+N P_{+},
\end{aligned}
$$

where K,L,M, and $\mathrm{N}$ are arbitrary constants. The infinitesimal symmetries are spanned by the four vector fields

$$
\begin{aligned}
& \mathbf{v}_{1}=\frac{\partial}{\partial x} \\
& \mathbf{v}_{2}=\frac{\partial}{\partial t} \\
& \mathbf{v}_{3}=P_{+} \frac{\partial}{\partial P_{+}} \\
& \mathbf{v}_{4}=v^{2} t \frac{\partial}{\partial x}+x \frac{\partial}{\partial t}-a x P_{+} \frac{\partial}{\partial P_{+}}
\end{aligned}
$$

with commutation relations

$$
\begin{aligned}
& {\left[\mathbf{v}_{1}, \mathbf{v}_{2}\right]=\left[\mathbf{v}_{1}, \mathbf{v}_{3}\right]=0, \quad\left[\mathbf{v}_{1}, \mathbf{v}_{4}\right]=\mathbf{v}_{2}-a \mathbf{v}_{3}} \\
& {\left[\mathbf{v}_{2}, \mathbf{v}_{3}\right]=0, \quad\left[\mathbf{v}_{2}, \mathbf{v}_{4}\right]=v^{2} \mathbf{v}_{1}, \quad\left[\mathbf{v}_{3}, \mathbf{v}_{4}\right]=0}
\end{aligned}
$$

We have ignored the obvious infinite dimensional subalgebras in the above analysis.

\section{CONCLUSION}

The main objective of this paper was to extend the method of deducing some fundamental linear partial differential equations of physics from a consideration of stochastic arguments to the nonlinear case. We saw that this could be achieved in a very simple way by modifying the master equation to obtain "nonlinear diffusion" equation, a "nonlinear Dirac equation" in 1 + 1 dimensions and the corresponding "non linear telegrapher's equation". As a preliminary step towards the analysis of the properties of the solutions, we have considered the group classification problem of the first and the last one by means of one parameter groups. The infinitesimal symmetry group of the nonlinear telegrapher's equation is spanned by a vector field corresponding to a "hyperbolic rotation" of $x$ and $t$. For our type of diffusion equation, though the group structure is similar to that of standard nonlinear diffusion equation, the 
ordinary differential equations obtained is different and the results are similar when $m=4$ in our case, but $m=-\frac{4}{3}$ in the standard case ( $m$ being the highest power of the dependent variable in coefficient to the $\frac{\partial^{2}}{\partial x^{2}}$ term in the nonlinear diffusion equation). The physical applications of this equation has been widely studied in the context of gas dynamics and plasma physics etc. We expect the other two equations to have similar important applications in physics with rich mathematical structure and we leave it for future study.However, as a comparison of equations (70) and (75) shows one does see explicitly which symmetries gets broken when the equation is modified.

\section{Acknowledgments}

The author is grateful to Prof. J. Ehlers for providing academic facilities at the Albert Einstein Institut, Potsdam, Germany, where this work was carried out. The author also wishes to thank S. Mahapatra for comments on the manuscript. 


\section{REFERENCES}

[1] Gaveau B, Jacobson T, Kac M and Schulman L S (1984) Phys. Rev. Lett. 53, 419

[2] Ord G N (1997) obtaining the Schrödinger and Dirac equations from the Einstein/Kac model of Brownian motion by projection Present status of the quantum theory of light ed. S. Jeffers etal (Dordrecht : Kluwer Academic)

[3] Ord G N (1997) Maxwell's equations directly from the dynamics of point particles Present status of the quantum theory of light ed. S. Jeffers etal (Dordrecht: Kluwer Academic)

[4] see e. g. Chandrasekhar S. (1943) Rev. Mod. Phys. 151

[5] McKeon D G C and Ord G N (1992) Phys. Rev. Lett. 69, 3

[6] Ovsiannikov L V (1982) Group analysis of differential equations (New York: Academic)

[7] Bluman G W and Cole J D (1969) Jour. of Math. and Mech. 181025

[8] Munier A, Burgan J R, Gutierrez J, Fijalkow E and Feix M R (1981) SIAM J. Appl. Math. 40191

[9] Hill J M (1982) Solutions of differential equations by one parameter groups (London : Pitman)

[10] Olver P J (1993) Application of groups to differential equations (Berlin : Springer) 